\title{
ULTRAHOLOMORPHIC SECTORIAL EXTENSIONS OF BEURLING TYPE
}

\author{
DAVID NICOLAS NENNING, ARMIN RAINER, AND GERHARD SCHINDL
}

\begin{abstract}
We prove sectorial extension theorems for ultraholomorphic function classes of Beurling type defined by weight functions with a controlled loss of regularity. The proofs are based on a reduction lemma, due to the second author, which allows to extract the Beurling from the Roumieu case, which was treated recently by Jiménez-Garrido, Sanz, and the third author. In order to have control on the opening of the sectors, where the extensions exist, we use the (mixed) growth index and the order of quasianalyticity of weight functions. As a consequence we obtain corresponding extension results for classes defined by weight sequences. Additionally, we give information on the existence of continuous linear extension operators.
\end{abstract}

\section{INTRODUCTION}

The aim of this work is to prove sectorial extension results of Borel-Ritt type. For a given formal power series with admissible growth behavior of the coefficients one looks for an ultraholomorphic function defined on a sector in the Riemann surface of the logarithm and asymptotic to the given series. Ultraholomorphic functions are holomorphic functions which satisfy certain growth conditions imposed on its iterated derivatives. In this paper we are primarily interested in the case that the growth conditions are defined by a weight function; in the spirit of Braun-MeiseTaylor classes 4. We allow for a controlled loss of regularity in the passage from formal power series to ultraholomorphic function. This manifests itself by the use of two weight functions and their mixed growth index which gives an upper bound on the opening of the sector on which the ultraholomorphic extension exists; abbreviating, we call this the mixed setting. Specifically, we treat the mixed Beurling case (for precise definitions see Section (3) by reducing it to the mixed Roumieu case which was investigated by Jiménez-Garrido, Sanz, and the third author in [11. This reduction procedure is based on a recent lemma proved and applied in a related context by the second author in [18].

Let us briefly recall the historic background on ultraholomorphic sectorial extensions. Classically, the problem was studied for Gevrey regularity, see Ramis 23]. Thilliez [30] generalized the results to suitable weight sequences $M$ and associated with $M$ a growth index $\gamma(M)$ which provides an upper bound for the opening of the sector on which the extension is defined. The paper of Thilliez also extends earlier results of Schmets and Valdivia 28. A different approach is pursued by Lastra, Malek, and Sanz [14, 15].

In the recent papers 8, 9, Jiménez-Garrido, Sanz, and the third author obtained analogous ultraholomorphic extension results for weight functions, by transferring the

Date: May 12, 2021.

2020 Mathematics Subject Classification. 26A12, 30D60, 46A13, 46E10 .

Key words and phrases. Ultraholomorphic function classes, extension results and extension operators, mixed setting, controlled loss of regularity, growth indices.

AR was supported by FWF-Project P 32905-N, DNN and GS by FWF-Project P 33417-N. 
"complex" method of [14, 15] as well as the "real" method of 30, respectively, and by exploiting the technique of associating a weight matrix with the given weight function, introduced in our article [19]. In analogy to $\gamma(M)$, a growth index $\gamma(\omega)$ with similar properties was associated with a weight function $\omega$.

In all these works no loss of regularity occurs in the extension procedure. The growth index $\gamma(\omega)$ is always dominated by the order of quasianalyticity $\mu(\omega)$; in general, one has the strict inequality $\gamma(\omega)<\mu(\omega)$, and the gap can be made arbitrarily large in individual examples. While $\gamma(\omega)$ is connected to the existence of extensions (on sectors of opening smaller than $\pi \gamma(\omega)$ ), the parameter $\mu(\omega)$ seems to be tied to the uniqueness of extensions (on sectors of opening larger than $\pi \mu(\omega)$ ). (The latter statement is confirmed for certain $\omega$ which admit a weight sequence description of the associated classes, but we conjecture that it holds in general.)

So, in order to have extensions on sectors of opening beyond $\pi \gamma(\omega)$, one is led to allow for a controlled loss of regularity: one weight function $\sigma$ measures the regularity of the formal power series, a second weight function $\omega$ that of its extension. The connection between $\sigma$ and $\omega$ is encoded in the mixed growth index $\gamma(\sigma, \omega)$, a natural generalization of $\gamma(\omega)$. In fact, extensions exist on all sectors of opening smaller than $\pi \gamma(\sigma, \omega)$. Since $\gamma(\omega) \leq \gamma(\sigma, \omega) \leq \mu(\omega)$, with generally strict inequalities, this means an improvement on the size of the sectors where extensions exist. Moreover, extensions on sectors of all openings smaller than $\pi \mu(\omega)$ exist if $\sigma$ is allowed to depend on the opening. These results were obtained in the Roumieu case by Jiménez-Garrido, Sanz, and the third author [11; analogous statements hold for weight sequences. It should be noted that, for technical reasons, the results involve a uniform shift of all weights (i.e., a multiplication by the sequence ( $p$ !) on the level of weight matrices) which here we ignored for simplicity. For a detailed study and comparison of the mentioned parameters we refer to [6.

The approach in [11] was the "complex" one of [8], since the "real" techniques of [9] failed in a crucial step. At the time of writing [11] the mixed Beurling case could not be handled. This changed thanks to a new reduction lemma proved by the second author in 18 in order to deal with a similar situation concerning the ultradifferentiable Whitney extension problem. Actually, this circle of ideas is intimately related to the problem at hand and was studied extensively in the literature. We refer the interested reader to the (by no means exhaustive) list of papers [1], [3], [5], [13], 28], 29], [10], [21, 22, [18.

In the setting of the present paper a weaker version of the aforementioned reduction lemma (namely, Lemma 4.4) suffices to fully reduce the Beurling to the Roumieu case. Thus it turns out that, again, the parameters $\gamma(\sigma, \omega)$ and $\mu(\omega)$ regulate the opening of the sectors on which extensions exist; see Theorem 4.1 and Theorem 4.6 In addition, we provide sufficient conditions for the existence of continuous linear extension operators on suitable subspaces.

We point out that the reduction procedure in [18] involves a small loss of information, since it leads to a stronger condition in the Beurling case. Thanks to the ramified nature of the mixed strong non-quasianalyticity condition defining the index $\gamma(\sigma, \omega)$, there is no loss of information in the ultraholomorphic sectorial extension problem.

The paper is organized as follows. After discussing weight functions and sequences in Section 2 and ultraholomorphic function and sequence spaces in Section 3 , we prove in Section 4 the main results on sectorial extension of mixed Beurling type for weight functions. In Theorem 4.1 the opening of the sector is controlled by $\gamma(\sigma, \omega)$ and in Theorem 4.6 by $\mu(\omega)$. In the final Section [5, the results for weight functions are applied to the case that the growth conditions are defined in terms of weight 
sequences, similarly using $\gamma(M, N)$ in Theorem 5.2 and $\mu(N)$ in Theorem 5.4. In all these theorems information on the existence of continuous linear extension operators is provided.

\section{WEIGHTS AND CONDITIONS}

2.1. Weight functions. A function $\omega:[0, \infty) \rightarrow[0, \infty)$ is called weight function if it is continuous, non-decreasing, $\omega(0)=0$, and $\lim _{t \rightarrow \infty} \omega(t)=\infty$. If in addition $\omega(t)=0$ for all $t \in[0,1]$, then $\omega$ is said to be normalized.

Let us consider the following (standardly used) conditions:

$\omega(2 t)=O(\omega(t))$ as $t \rightarrow \infty$. $\omega(t)=O(t)$ as $t \rightarrow \infty$. $\log (t)=o(\omega(t))$ as $t \rightarrow \infty$. $\varphi_{\omega}: t \mapsto \omega\left(e^{t}\right)$ is a convex function on $\mathbb{R}$.

$\left(\omega_{\mathrm{nq}}\right)$ $\omega(t)=o(t)$ as $t \rightarrow \infty$. $\exists H \geq 1 \forall t \geq 0: 2 \omega(t) \leq \omega(H t)+H$.

Weight functions $\omega$ satisfying $\left(\omega_{\mathrm{nq}}\right)$ are said to be non-quasianalytic and those satisfying $\left(\omega_{\mathrm{snq}}\right)$ are called strongly non-quasianalytic or simply strong. Note that $\left(\omega_{\mathrm{snq}}\right)$ $\Rightarrow\left(\omega_{\mathrm{nq}}\right) \Rightarrow\left(\omega_{5}\right) \Rightarrow\left(\omega_{2}\right)$.

For ease of reference we define the following sets of weight functions:

$$
\begin{aligned}
\mathcal{W}_{0} & :=\left\{\omega: \omega \text { is a normalized weight function satisfying }\left(\omega_{3}\right) \text { and }\left(\omega_{4}\right)\right\}, \\
\mathcal{W} & :=\left\{\omega \in \mathcal{W}_{0}: \omega \text { satisfies }\left(\omega_{1}\right)\right\} .
\end{aligned}
$$

We remark that in 18 the elements of $\mathcal{W}$ (and only those) were called (normalized) weight functions.

For any $\omega \in \mathcal{W}_{0}$ we define the Young conjugate of $\varphi_{\omega}$ by

$$
\varphi_{\omega}^{*}(x):=\sup \left\{x y-\varphi_{\omega}(y): y \geq 0\right\}, \quad x \geq 0 ;
$$

it will appear in Lemma 2.3 and in the definition of the ultraholomorphic classes in Section 3 .

Given two weights $\sigma, \tau$ we write $\sigma \preceq \tau$ if $\tau(t)=O(\sigma(t))$ as $t \rightarrow \infty$; it reflects the inclusion relation of the corresponding ultraholomorphic classes, see Section 3.4. We call two weights $\sigma$ and $\tau$ equivalent if $\sigma \preceq \tau$ and $\tau \preceq \sigma$.

2.2. Weight functions obtained by power substitutions. For any weight function $\omega$ and $r>0$ we denote by $\omega^{r}$ the weight $\omega^{r}(t):=\omega\left(t^{r}\right)$ resulting from the power substitution $t \mapsto t^{r}$. Clearly, $\left(\omega^{r}\right)^{s}=\omega^{r s}$ for any $r, s>0$.

It is easy to see (cf. [11, p.1635]) that $\omega^{r} \in \mathcal{W}$ if and only if $\omega \in \mathcal{W}$. Furthermore, we have $o \preceq r$ if and only if $\sigma^{r} \preceq r^{r}$. In particular, $\sigma$ and $\tau$ are equivalent if and only if $\sigma^{r}$ and $\tau^{r}$ are equivalent (for some/any $r>0$ ).

On the other hand, $\left(\omega_{\mathrm{nq}}\right),\left(\omega_{\mathrm{snq}}\right)$, and $\left(\omega_{5}\right)$ might, in general, not be preserved when passing from $\omega$ to $\omega^{r}$. In fact (cf. [11, (1)]), for $r>0$ the weight function $\omega^{r}$ is non-quasianalytic (i.e., satisfies $\left(\omega_{\mathrm{nq}}\right)$ ) if and only if $\omega$ fulfills

$$
\left(\omega_{\mathrm{nq}_{r}}\right) \quad \int_{1}^{\infty} \frac{\omega(t)}{t^{1+1 / r}} d t<\infty .
$$


2.3. Mixed growth index. For weight functions $\omega, \sigma$ and $r>0$ we recall the condition (cf. [11, Section 3.1] and also [10, (5.4)])

$$
(\sigma, \omega)_{\gamma_{r}} \quad \exists C>0 \forall t \geq 0: \int_{1}^{\infty} \frac{\omega(t y)}{y^{1+1 / r}} d y \leq C \sigma(t)+C .
$$

Note that, $\omega$ being non-decreasing, the integral is bounded below by $r \omega(t)$ so that this condition implies $o$ 飞 $\omega$. Clearly, $(\sigma, \omega)_{\gamma_{r}}$ implies $(\sigma, \omega)_{\gamma_{r^{\prime}}}$ for all $0<r^{\prime}<r$.

The mixed growth index is defined by

$$
\gamma(\sigma, \omega):=\sup \left\{r>0:(\sigma, \omega)_{\gamma_{r}} \text { is satisfied }\right\}
$$

and $\gamma(\sigma, \omega):=0$ if $(\sigma, \omega)_{\gamma_{r}}$ holds for no $r>0$. Putting $\gamma(\omega):=\gamma(\omega, \omega)$ we recover the growth index $\gamma(\omega)$ introduced and studied in [6, 8, 9,

We have $\gamma(\omega) \leq \gamma(\sigma, \omega)$ provided that $o \underline{ }, \omega$, cf. [11, Lemma 3]. By 6, Corollary 2.14], $\gamma(\omega)>0$ if and only if ( $\left.\omega_{1}\right)$ holds true. In particular, for $\left.\omega \in \mathcal{W}\right] \gamma(\sigma, \omega)>0$ if and only if $o \preceq \omega$.

A weight function $\omega$ is strongly non-quasianalytic (i.e. satisfies $\left(\omega_{\text {snq }}\right)$ ) if and only if $\gamma(\omega)>1$; see [6. Corollary 2.13]. And, clearly, $\gamma(\sigma, \omega)>1$ implies that $\omega$ is non-quasianalytic and thus satisfies $\left(\omega_{5}\right)$.

Note that $(\sigma, \omega)_{\gamma_{r}}$ if and only if $\left(\sigma^{r}, \omega^{r}\right)_{\gamma_{1}}$ and so (cf. [11, Remark $\left.7(i)\right]$ )

$$
\gamma(\sigma, \omega)=r \gamma\left(\sigma^{r}, \omega^{r}\right) \quad \text { for all } r>0 .
$$

Remark 2.1. In [18] the condition $(\sigma, \omega)_{\gamma_{1 / r}}$ was denoted by $\left(S_{r}\right)$ and the pair $(\omega, \sigma)$ was called $1 / r$-strong.

2.4. Order of quasianalyticity. The order of quasianalyticity of a weight function $\omega$ is defined by (cf. [11, (18)])

$$
\mu(\omega):=\sup \left\{r>0: \int_{1}^{\infty} \frac{\omega(u)}{u^{1+1 / r}} d u<\infty\right\}=\sup \left\{r>0: \omega \text { satisfies }\left(\omega_{\mathrm{nq}_{r}}\right)\right\}
$$

and $\mu(\omega):=0$ if $\left(\omega \omega_{\mathrm{nq}_{\mathrm{r}}}\right)$ holds for no $r>0$. It is preserved under equivalence of weight functions, since the condition $\left(\omega \omega_{\mathrm{nq}_{r}}\right)$ is preserved.

We have $\gamma(\sigma, \omega) \leq \mu(\omega)$ for any weight $\sigma \preceq \mu \omega$, cf. [11, Lemma 7]. Thus $\mu(\omega)>0$ if $\omega \in \mathcal{W}$, by the properties of the mixed growth index, see Section 2.3 .

2.5. Weight sequences. Any positive sequence $M=\left(M_{p}\right) \in \mathbb{R}_{>0}^{\mathbb{N}}$ is called weight sequence. With $M$ we associate the sequences $m=\left(m_{p}\right)$ and $\mu=\left(\mu_{p}\right)$ defined by $m_{p}:=\frac{M_{p}}{p !}$ and $\mu_{p}:=\frac{M_{p}}{M_{p-1}}, \mu_{0}:=1$, respectively. A weight sequence $M$ is called normalized if $1=M_{0} \leq M_{1}$. For any weight sequence $M$ and $r>0$ we define the power $M^{r}:=\left(\left(M_{p}\right)^{r}\right)_{p \in \mathbb{N}}$.

A weight sequence $M$ is called log-convex if

$$
\forall p \in \mathbb{N}_{>0}: M_{p}^{2} \leq M_{p-1} M_{p+1},
$$

which is equivalent to $\mu$ being non-decreasing. It is called strongly log-convex if (IC) holds for the associated sequence $m$. We say that $M$ has moderate growth if

$$
\exists C \geq 1 \forall p, q \in \mathbb{N}: M_{p+q} \leq C^{p+q} M_{p} M_{q} .
$$

Replacing $M$ by $m$ or by $M^{r}$ (for arbitrary $r>0$ ) gives an equivalent condition. A weight sequence $M$ is called non-quasianalytic, if

$$
\sum_{p=1}^{\infty} \frac{1}{\mu_{p}}<\infty
$$


Note that $M^{1 / r}$ is non-quasianalytic if and only if $M$ satisfies

$\left(\mathrm{nq}_{r}\right)$

$$
\sum_{p=1}^{\infty}\left(\frac{1}{\mu_{p}}\right)^{1 / r}<\infty .
$$

For later reference we consider the conditions (cf. [25], [17] and [2])

$$
\begin{gathered}
\sup _{p \in \mathbb{N}_{>0}} \frac{\mu_{p}}{p} \sum_{k \geq p} \frac{1}{\mu_{k}}<\infty, \\
\exists Q \in \mathbb{N}_{>0}: \liminf _{p \rightarrow \infty} \frac{\mu_{Q p}}{\mu_{p}}>1 .
\end{gathered}
$$

Two weight sequences $M$ and $N$ are said to be equivalent if $C^{-1} \leq\left(\frac{M_{p}}{N_{p}}\right)^{1 / p} \leq C$ for some $C>0$ (cf. Section 3.4).

For ease of reference we introduce the set of weight sequences

$$
\mathcal{L C}:=\left\{M \in \mathbb{R}_{>0}^{\mathbb{N}}: M \text { is normalized, log-convex, } \lim _{p \rightarrow \infty}\left(M_{p}\right)^{1 / p}=\infty\right\} .
$$

We shall recall the (mixed) growth index and the order of quasianalyticity for weight sequences in Section 5 .

2.6. Associated function. With $M \in[\mathcal{L C}$ one associates (cf. [16, Chapitre I] and [12, Definition 3.1]) the function $\omega_{M}:[0, \infty) \rightarrow[0, \infty)$ defined by

$$
\omega_{M}(t):=\sup _{p \in \mathbb{N}} \log \left(\frac{t^{p}}{M_{p}}\right) \text { for } t>0, \quad \omega_{M}(0):=0 .
$$

An easy calculation shows that, for all $r>0$,

$$
\omega_{M}^{r}=r \omega_{M^{1 / r}} .
$$

We collect some well-known properties for $\omega_{M}$.

Lemma 2.2 (Cf. 8, Lem. 2.4] and [9, Lem. 3.1]). Let $M \in \mathcal{L C}$. Then:

(i) $\omega_{M}$ belongs to $\mathcal{W}_{0}$

(ii) If $M$ satisfies $\left(\sqrt{\gamma_{1}}\right)$, then $\omega_{M}$ fulfills $\left(\omega_{\mathrm{snq}}\right)$ (which in turn implies $\left(\omega_{1}\right)$ ).

(iii) $M$ has moderate growth if and only if $\omega_{M}$ satisfies ( $\left.\omega_{6}\right)$.

2.7. Weight matrices. Cf. [19, Section 4]. A weight matrix $\mathcal{M}$ is a (one parameter) family of weight sequences $\mathcal{M}:=\left\{M^{[x]}: x \in \mathbb{R}_{>0}\right\}$ such that each $M^{[x]}$ is normalized and non-decreasing, and $M^{[x]} \leq M^{[y]}$ if $x \leq y$. We call a weight matrix $\mathcal{M}$ standard log-convex, abbreviated by $\left(\mathcal{M}_{\mathrm{sc}}\right)$, if $M^{[x]} \in \mathcal{L C}$ for all $x>0$.

Weight matrices are a convenient technical tool for working with weight functions:

Lemma 2.3 ([19, Section 5]). With every $\omega \in \mathcal{W}_{0}$ one can associate an $\left.\left(\mathcal{M}_{\mathrm{sc}}\right)\right]$ weight matrix $\Omega:=\left\{W^{[l]}: l>0\right\}$ by setting

$$
W_{j}^{[l]}:=\exp \left(\frac{1}{l} \varphi_{\omega}^{*}(l j)\right)
$$

If $\omega$ additionally satisfies $\left(\omega_{1}\right)$, then

$$
\forall h \geq 1 \exists A \geq 1 \forall l>0 \exists D \geq 1 \forall j \in \mathbb{N}: h^{j} W_{j}^{[l]} \leq D W_{j}^{[A l]} .
$$

Moreover, $\omega \in \mathcal{W}_{0}$ is non-quasianalytic if and only if some/each $W^{[l]}$ is nonquasianalytic. All weight sequences $W^{[l]}$ are equivalent if and only if $\omega$ satisfies (w) which in turn is equivalent to some/each $W^{[l]}$ having moderate growth. 


\section{Ultraholomorphic FUnCTION Classes AND the Borel MaP}

We recall definitions and basic facts on ultraholomorphic classes; cf. 9, Section 2.5], [11, Section 2.7], 28], 30, and references therein.

3.1. Sectors. Let $\mathcal{R}$ be the Riemann surface of the logarithm. We wish to work in general unbounded open sectors in $\mathcal{R}$ with vertex at 0 , but all our results will be unchanged under rotation. So it suffices to consider unbounded open sectors

$$
S_{\gamma}:=\left\{z \in \mathcal{R}:|\arg (z)|<\frac{\gamma \pi}{2}\right\}, \quad \gamma>0,
$$

of opening $\gamma \pi$ bisected by the positive real axis; we refer to them simply as sectors.

3.2. Ultraholomorphic classes associated with a weight sequence. Let $M$ be a weight sequence, $S$ a sector, and $h>0$. We consider the Banach space

$$
\mathcal{A}_{M, h}(S):=\left\{f \in \mathcal{H}(S): \sup _{z \in S, p \in \mathbb{N}} \frac{\left|f^{(p)}(z)\right|}{h^{p} M_{p}}<\infty\right\},
$$

where $\mathcal{H}(S)$ is the space of holomorphic functions on $S$. We define the spaces

$$
\mathcal{A}_{(M)}(S):=\bigcap_{h>0} \mathcal{A}_{M, h}(S) \quad \text { and } \quad \mathcal{A}_{\{M\}}(S):=\bigcup_{h>0} \mathcal{A}_{M, h}(S)
$$

and equip them with their natural locally convex topologies. The Fréchet space $\mathcal{A}_{(M)}(S)$ is the ultraholomorphic class of Beurling type, the (LB) space $\mathcal{A}_{\{M\}}(S)$ the ultraholomorphic class of Roumieu type associated with $M$ in the sector $S$.

Analogously we introduce the sequence spaces

$$
\begin{aligned}
& \Lambda_{M, h}:=\left\{a=\left(a_{p}\right) \in \mathbb{C}^{\mathbb{N}}: \sup _{p \in \mathbb{N}} \frac{\left|a_{p}\right|}{h^{p} M_{p}}<\infty\right\}, \\
& \Lambda_{(M)}:=\bigcap_{h>0} \Lambda_{M, h}, \quad \text { and } \quad \Lambda_{\{M\}}:=\bigcup_{h>0} \Lambda_{M, h} .
\end{aligned}
$$

We have the (asymptotic) Borel maps $\mathcal{B}: \mathcal{A}_{(M)}(S) \rightarrow \Lambda_{(M)}$ and $\mathcal{B}: \mathcal{A}_{\{M\}}(S) \rightarrow \Lambda_{\{M\}}$ given by $f \mapsto\left(f^{(p)}(0)\right)_{p \in \mathbb{N}}$, where $f^{(p)}(0):=\lim _{z \in S, z \rightarrow 0} f^{(p)}(z)$.

3.3. Ultraholomorphic classes associated with a weight function. Let $\omega$ be a normalized weight function satisfying $\left(\omega_{3}\right)$. For a sector $S$ and $l>0$, we have the Banach space

$$
\mathcal{A}_{\omega, l}(S):=\left\{f \in \mathcal{H}(S): \sup _{z \in S, p \in \mathbb{N}} \frac{\left|f^{(p)}(z)\right|}{\exp \left(\frac{1}{l} \varphi_{\omega}^{*}(l p)\right)}<\infty\right\} .
$$

We define the spaces

$$
\mathcal{A}_{(\omega)}(S):=\bigcap_{l>0} \mathcal{A}_{\omega, l}(S) \quad \text { and } \quad \mathcal{A}_{\{\omega\}}(S):=\bigcup_{l>0} \mathcal{A}_{\omega, l}(S)
$$

and equip them with their natural locally convex topologies. The Fréchet space $\mathcal{A}_{(\omega)}(S)$ is the ultraholomorphic class of Beurling type, the (LB) space $\mathcal{A}_{\{\omega\}}(S)$ the ultraholomorphic class of Roumieu type associated with $\omega$ in the sector $S$.

Correspondingly, we have the sequence spaces

$$
\begin{aligned}
& \Lambda_{\omega, l}:=\left\{a=\left(a_{p}\right) \in \mathbb{C}^{\mathbb{N}}: \sup _{p \in \mathbb{N}} \frac{\left|a_{p}\right|}{\exp \left(\frac{1}{l} \varphi_{\omega}^{*}(l p)\right)}<\infty\right\}, \\
& \Lambda_{(\omega)}:=\bigcap_{l>0} \Lambda_{\omega, l}, \quad \text { and } \quad \Lambda_{\{\omega\}}:=\bigcup_{l>0} \Lambda_{\omega, l} .
\end{aligned}
$$

We get the Borel maps $\mathcal{B}: \mathcal{A}_{(\omega)}(S) \rightarrow \Lambda_{(\omega)}$ and $\mathcal{B}: \mathcal{A}_{\{\omega\}}(S) \rightarrow \Lambda_{\{\omega\}}$. 
For a weight matrix $\mathcal{M}=\left\{M^{[x]}: x>0\right\}$ and a sector $S$ we define ultraholomorphic classes of Beurling and Roumieu type

$$
\mathcal{A}_{(\mathcal{M})}(S):=\bigcap_{x>0} \mathcal{A}_{\left(M^{[x]}\right)}(S), \quad \mathcal{A}_{\{\mathcal{M}\}}(S):=\bigcup_{x>0} \mathcal{A}_{\left\{M^{[x]}\right\}}(S),
$$

as well as sequence spaces

$$
\Lambda_{(\mathcal{M})}:=\bigcap_{x>0} \Lambda_{(M[x])}, \quad \Lambda_{\{\mathcal{M}\}}:=\bigcup_{x>0} \Lambda_{\left\{M^{[x]}\right\}},
$$

and equip them with their natural locally convex topologies. Clearly, we have the associated Borel maps.

Proposition 3.1. Let $\omega \in \mathcal{W}$ and let $\Omega$ be the associated weight matrix. Then

$$
\mathcal{A}_{(\omega)}(S)=\mathcal{A}_{(\Omega)}(S), \quad \mathcal{A}_{\{\omega\}}(S)=\mathcal{A}_{\{\Omega\}}(S), \quad \Lambda_{(\omega)}=\Lambda_{(\Omega)}, \quad \Lambda_{\{\omega\}}=\Lambda_{\{\Omega\}},
$$

as locally convex vector spaces.

Proof. This is a consequence of [19, Lemma 5.9, (5.10)] (see (4)) and the way how the seminorms are defined in these spaces.

3.4. Inclusion relations. As an immediate consequence of the definitions we get the following inclusion relations (on any sector):

- $\sup _{p \in \mathbb{N}_{>0}}\left(\frac{M_{p}}{N_{p}}\right)^{1 / p}<\infty$ implies the inclusions $\mathcal{A}_{(M)} \subseteq \mathcal{A}_{(N)}, \mathcal{A}_{\{M\}} \subseteq \mathcal{A}_{\{N\}}$, $\Lambda_{(M)} \subseteq \Lambda_{(N)}$, and $\Lambda_{\{M\}} \subseteq \Lambda_{\{N\}}$

- $\left(\frac{M_{p}}{N_{p}}\right)^{1 / p} \rightarrow 0$, abbreviated by $M \triangleleft N$, implies $\mathcal{A}_{\{M\}} \subseteq \mathcal{A}_{(N)}$ and $\Lambda_{\{M\}} \subseteq$ $\Lambda_{(N)}$.

- $\tau(t)=O(\sigma(t))$ as $t \rightarrow \infty$ (i.e. $\sigma \preceq \tau)$ implies $\mathcal{A}_{(\sigma)} \subseteq \mathcal{A}_{(\tau)}, \mathcal{A}_{\{\sigma\}} \subseteq \mathcal{A}_{\{\tau\}}$, $\Lambda_{(\sigma)} \subseteq \Lambda_{(\tau)}$, and $\Lambda_{\{\sigma\}} \subseteq \Lambda_{\{\tau\}}$.

- $\tau(t)=o(\sigma(t))$ as $t \rightarrow \infty$ implies $\mathcal{A}_{\{\sigma\}} \subseteq \mathcal{A}_{(\tau)}$ and $\Lambda_{\{\sigma\}} \subseteq \Lambda_{(\tau)}$.

Obviously, we have $\mathcal{A}_{*}\left(S_{r}\right) \subseteq \mathcal{A}_{*}\left(S_{r^{\prime}}\right)$ for any $0<r^{\prime} \leq r$, where $*$ refers to any of the specified regularity classes. All listed inclusions are continuous.

\section{UltraholomorPhiC SECTORIAL EXTENSIONS}

In this section we prove the main Theorems 4.1 and 4.6. The proof of Theorem 4.1 is based on Lemma 4.4 which allows us to reduce the Beurling case to the Roumieu case (treated in [11] and recalled in Theorems 4.2 and 4.3). Theorem 4.6] is a corollary of Theorem 4.1. We work with two weight functions $\omega$ and $\sigma$ allowing for a controlled loss of regularity in the extension procedure. This generalizes the case $\omega=\sigma$ treated in [9, Section 7].

In the following, by an extension operator we mean a continuous linear right-inverse of the Borel map $\mathcal{B}$; the domain and codomain will be clear from the context.

\subsection{Notation for associated weights.}

- For any weight function $\omega \in \mathcal{W}_{0}$ we denote by $\mathcal{M}(\omega)$ the weight matrix $\left\{W^{[l]}: l>0\right\}$ associated with $\omega$ in Lemma 2.3 .

- For any weight matrix $\mathcal{M}=\left\{M^{[x]}: x>0\right\}$ consider the weight matrix $\widehat{\mathcal{M}}=\left\{\widehat{M}^{[x]}: x>0\right\}$, where $\widehat{M}_{p}^{[x]}:=p ! M_{p}^{[x]}$ for all $p \in \mathbb{N}$.

- For a weight matrix $\mathcal{M}=\left\{M^{[x]}: x>0\right\}$ satisfying $\left(\mathcal{M}_{\mathrm{sc}}\right) \operatorname{set} \omega(\widehat{\mathcal{M}}):=\omega_{\widehat{M}^{[1]}}$.

Then $\omega(\widehat{\mathcal{M}}) \in \mathcal{W}$ and for all $\tau \in[\mathcal{W}$ equivalent to $\omega(\widehat{\mathcal{M}})$ we have topological isomorphisms $\mathcal{A}_{\{\tau\}}(S) \cong \mathcal{A}_{\{\widehat{\mathcal{M}}\}}(S)$ and $\mathcal{A}_{(\tau)}(S) \cong \mathcal{A}_{(\widehat{\mathcal{M}})}(S)$ for all sectors $S$, analogously $\Lambda_{\{\tau\}} \cong \Lambda_{\{\widehat{\mathcal{M}}\}}$ and $\Lambda_{(\tau)}=\Lambda_{(\widehat{\mathcal{M}})}$; see [8, Theorem 5.3] and [9, Theorem 6.7] (the proof 
is based on [26, Corollary $3.17(i i) \Rightarrow(i)])$. We point out that $\omega_{\widehat{M}^{[x]}}$ is equivalent to $\omega(\widehat{\mathcal{M}})$ for all $x>0$; see [8, Lemma 5.1, Cor. 5.2, Thm. 5.3].

Note that for $\omega(\mathcal{M}):=\omega_{M^{[1]}}$ condition $\left(\underline{\omega_{1}}\right)$ and the above properties might fail.

4.2. Extensions of Beurling type controlled by the mixed growth index. The main goal of this section is to prove the following theorem. Recall that, for $\omega \in \mathcal{W}, \gamma(\sigma, \omega)>0$ if and only if $o \underline{\sigma} \omega$ (see Section 2.3).

Theorem 4.1. Let $\omega, \sigma \in \mathcal{W}$ and $0<\gamma<\gamma(\sigma, \omega)$. Consider $\tau_{1}:=\omega(\widehat{\mathcal{M}(\sigma)})$ and $\tau_{2}:=\omega(\widehat{\mathcal{M}(\omega)})$. Then:

(i) We have the inclusion $\mathcal{B}\left(\mathcal{A}_{\left(\tau_{2}\right)}\left(S_{\gamma}\right)\right) \supseteq \Lambda_{\left(\tau_{1}\right)}$.

(ii) If $\tau \in \mathcal{W}$ satisfies $\sigma(t)=o(\tau(t))$ as $t \rightarrow \infty$, then there exists an extension operator

$$
\mathcal{E}^{\tau_{3}, \tau_{2}}: \Lambda_{\left\{\tau_{3}\right\}} \rightarrow \mathcal{A}_{\left(\tau_{2}\right)}\left(S_{\gamma}\right),
$$

where $\tau_{3}:=\omega(\widehat{\mathcal{M}(\tau)})$.

The proof is based on a reduction to the Roumieu case which will be now recalled.

\subsection{Extensions of Roumieu type controlled by the mixed growth index.}

Theorem 4.2 ([11, Theorem 2]). Let $\sigma$ and $\omega$ be normalized weight functions satisfying (w3). Assume that $\gamma(\sigma, \omega)>0$ and let $0<\gamma<\gamma(\sigma, \omega)$. Consider the weight matrices $\Sigma=\left\{S^{[x]}: x>0\right\}:=\mathcal{M}(\sigma), \Omega=\left\{W^{[x]}: x>0\right\}:=\mathcal{M}(\omega)$, as well as $\widehat{\Sigma}$ and $\widehat{\Omega}$. Then there exists a constant $k_{0}>0$ such that for every $x>0$ and every $h>0$ we have an extension operator

$$
\mathcal{E}_{h}^{\sigma, \omega}: \Lambda_{\widehat{S}[x], h} \rightarrow \mathcal{A}_{\widehat{W}[8 x], k_{0} h}\left(S_{\gamma}\right) .
$$

Consequently, we have the inclusion $\mathcal{B}\left(\mathcal{A}_{\{\widehat{\Omega}\}}\left(S_{\gamma}\right)\right) \supseteq \Lambda_{\{\widehat{\Sigma}\}}$.

Theorem 4.3 ([11, Corollary 1]). Let $\sigma, \omega \in \mathcal{W}$ and $0<\gamma<\gamma(\sigma, \omega)$. Consider $\tau_{1}:=\omega(\widehat{\mathcal{M}(\sigma)})$ and $\tau_{2}:=\omega(\widehat{\mathcal{M}(\omega)})$. Then for every $l>0$ there exist $l_{1}>0$ and an extension operator

$$
\mathcal{E}_{l}^{\tau_{1}, \tau_{2}}: \Lambda_{\tau_{1}, l} \rightarrow \mathcal{A}_{\tau_{2}, l_{1}}\left(S_{\gamma}\right) .
$$

In particular, we have $\mathcal{B}\left(\mathcal{A}_{\left\{\tau_{2}\right\}}\left(S_{\gamma}\right)\right) \supseteq \Lambda_{\left\{\tau_{1}\right\}}$.

4.4. Reduction lemma. The reduction is based on the following variant of 18 Lemma 13] (which in turn contains ideas from [1, Lemma 4.4]). The proof simplifies significantly, because in contrast to [18] we need not bother about concavity of the weights.

Lemma 4.4. Let $\omega, \sigma$ be (normalized) weight functions such that $\gamma(\sigma, \omega)>1$. Let $f:[0, \infty) \rightarrow[0, \infty)$ satisfy $\sigma(t)=o(f(t))$ as $t \rightarrow \infty$. Then there exist (normalized) weight functions $\widetilde{\omega}, \widetilde{\sigma}$ satisfying $\gamma(\widetilde{\sigma}, \widetilde{\omega})>1$ and

$$
\omega(t)=o(\widetilde{\omega}(t)), \quad \sigma(t)=o(\widetilde{\sigma}(t)), \quad \widetilde{\sigma}(t)=o(f(t)) \quad \text { as } t \rightarrow \infty .
$$

If $\omega, \sigma \in \mathcal{\mathcal { W } _ { 0 }}$ (resp. $\omega, \sigma \in \mathcal{W}$ ), then we may assume that also $\widetilde{\omega}, \widetilde{\sigma} \in \mathcal{\mathcal { W } _ { 0 }}$ (resp. $\widetilde{\omega}, \widetilde{\sigma}(\mathcal{W})$.

Proof. By [18, Proposition 7], the condition $\gamma(\sigma, \omega)>1$ is equivalent to

$$
\exists C>0 \exists K>H>1 \exists t_{0} \geq 0 \forall t \geq t_{0} \forall j \in \mathbb{N}_{>0}: \omega\left(K^{j} t\right) \leq C H^{j} \sigma(t) .
$$

We will construct weight functions $\widetilde{\omega}$ and $\widetilde{\sigma}$ satisfying (5) and (6), i.e., $\gamma(\widetilde{\sigma}, \widetilde{\omega})>1$. 
Note that $f(t) \rightarrow \infty$ as $t \rightarrow \infty$, since $\sigma(t) \rightarrow \infty$ and $\sigma(t)=o(f(t))$ as $t \rightarrow \infty$. We consider a strictly increasing sequence $\left(x_{n}\right)_{n \geq 1}$ tending to infinity, with $x_{1}:=0$, $x_{2} \geq 1$, and satisfying the following requirements for all $n \geq 2$ :

$$
\begin{aligned}
x_{n} & >\max \{2, K\} x_{n-1}+n, \\
f(t) & \geq n^{2} \sigma(t), \quad \text { for all } t \geq x_{n}, \\
\omega\left(x_{n}\right) & \geq 2^{n-i} \omega\left(x_{i}\right), \quad \text { for all } 1 \leq i \leq n-1, \\
\sigma\left(x_{n}\right) & \geq 2^{n-i} \sigma\left(x_{i}\right), \quad \text { for all } 1 \leq i \leq n-1 .
\end{aligned}
$$

Then we define the weights $\widetilde{\omega}$ and $\widetilde{\sigma}$ as follows: for $n \geq 1$ and $t \in\left[x_{n}, x_{n+1}\right)$ set

$$
\widetilde{\omega}(t):=n \omega(t)-\sum_{i=1}^{n} \omega\left(x_{i}\right) \quad \text { and } \quad \widetilde{\sigma}(t):=n \sigma(t)-\sum_{i=1}^{n} \sigma\left(x_{i}\right) .
$$

By definition and since $x_{2} \geq 1, \widetilde{\omega}$ is normalized if $\omega$ is normalized; analogously for $\widetilde{\sigma}$. Moreover, both $\widetilde{\omega}$ and $\widetilde{\sigma}$ are non-decreasing, continuous, tending to infinity as $t \rightarrow \infty$, and vanish at 0 . Note that $\widetilde{\omega}$ satisfies $\left(\omega_{4}\right)$ provided that $\omega$ does; similarly for $\widetilde{\sigma}$.

As in [18, Lemma 13] one shows that, for all $n \geq 2$ and all $t \in\left[x_{n}, x_{n+1}\right)$,

$$
\begin{aligned}
(n-2) \omega(t) & \leq \widetilde{\omega}(t) \leq n \omega(t), \\
(n-2) \sigma(t) & \leq \widetilde{\sigma}(t) \leq n \sigma(t) .
\end{aligned}
$$

Consequently, $\omega(t)=o(\widetilde{\omega}(t))$ and $\sigma(t)=o(\widetilde{\sigma}(t))$ as $t \rightarrow \infty$. In particular, $\widetilde{\omega}$ satisfies $\left(\omega_{3}\right)$ provided that $\omega$ does; similarly for $\widetilde{\sigma}$. Hence $\widetilde{\omega}, \widetilde{\sigma} \in \mathcal{W}_{0}$ provided that $\omega, \sigma \in \mathcal{W}_{0}$ Combining (8) and (12) yields $\widetilde{\sigma}(t)=o(f(t))$ as $t \rightarrow \infty$. So also (5) is shown.

Now $\gamma(\widetilde{\omega}, \widetilde{\sigma})>1$ follows from (6), (7), (11), and (12) as in the proof of 18, Lemma 13]. By a similar argument (see loc. cit.), $\widetilde{\omega}, \widetilde{\sigma}$ satisfy $\left(\omega_{1}\right)$ if $\omega, \sigma$ do so.

We also need the following observation.

Lemma 4.5. Let $\omega, \sigma \in \mathcal{W}$ satisfy $\omega(t)=o(\sigma(t))$ as $t \rightarrow \infty$, and consider $\Omega=$ $\left\{W^{[x]}: x>0\right\}:=\mathcal{M}(\omega)$ and $\Sigma=\left\{S^{[x]}: x>0\right\}:=\mathcal{M}(\sigma)$. Then

$$
\forall H>0 \forall x>0 \exists C>0: \widehat{S}^{[x]} \leq C \widehat{W}^{[H x]} .
$$

Proof. [19, Lemma 5.16] implies

$$
\forall H>0 \forall x>0 \exists C>0: S^{[x]} \leq C W^{[H x]} .
$$

which is obviously equivalent to the assertion.

4.5. Proof of Theorem 4.1. (i) The argument follows a well-known scheme used, e.g., in the proofs of [1, Theorem 4.5], [9, Theorem 7.2], and [18, Theorem 2].

Fix $r>0$ such that $\gamma<r<\gamma(\sigma, \omega)$. We consider the weight functions $\omega^{r}, \sigma^{r} \in \mathcal{W}$ which satisfy $\gamma\left(\sigma^{r}, \omega^{r}\right)>1$, by (2).

Set $\Sigma:=\mathcal{M}(\sigma)$ and $\Omega:=\mathcal{M}(\omega)$. Let $\widehat{a}=\left(\widehat{a}_{p}\right) \in \Lambda_{\left(\tau_{1}\right)}=\Lambda_{(\widehat{\Sigma})}$ be given. Our goal is to show that $\widehat{a} \in \mathcal{B}\left(\mathcal{A}_{(\widehat{\Omega})}\left(S_{\gamma}\right)\right)=\mathcal{B}\left(\mathcal{A}_{\left(\tau_{2}\right)}\left(S_{\gamma}\right)\right)$.

To this end we consider $a:=\left(a_{p}\right)=\left(\widehat{a}_{p} / p !\right)$ and the function

$$
g(t):=\log \max \left\{1,\left|a_{p}\right|\right\}, \quad p \leq t<p+1, p \in \mathbb{N} .
$$

Since $a \in \Lambda_{(\Sigma)}=\Lambda_{(\sigma)}$, for each integer $j \geq 1$ there exists $C_{j}>0$ such that

$$
g(t) \leq j \varphi_{\sigma}^{*}(t / j)+C_{j}, \quad \text { for all } t \geq 0 .
$$

Using [1, Lemma 4.3] (for $\psi_{j}:=j \varphi_{\sigma}^{*}(t / j)$ ), we conclude that there is a convex function $h:[0, \infty) \rightarrow[0, \infty)$ and a positive sequence $\left(D_{j}\right)_{j}$ such that

$$
g \leq h \leq \inf _{j \geq 1}\left(j \varphi_{\sigma}^{*}(t / j)+D_{j}\right) .
$$


(In this step we need $\left(\omega_{1}\right)$ for $\sigma$ in order to assume w.l.o.g. that $\sigma$, and hence $\varphi_{\sigma}$, is of class $\mathcal{C}^{1}$, and $\varphi_{\sigma}^{\prime}(t) \rightarrow \infty$ as $t \rightarrow \infty$. Thus $\varphi_{\sigma}^{*}$ is differentiable and $\left(\varphi_{\sigma}^{*}\right)^{\prime}=\left(\varphi_{\sigma}^{\prime}\right)^{-1}$. See [4, p.210], [18, Lemma 15] and also [1, Theorem 4.5].)

Then the Young conjugate $h^{*}$ of $h$ satisfies

$$
h^{*}(t) \geq j \varphi_{\sigma}(t)-D_{j}, \quad \text { for all } t \text { and all } j,
$$

and thus

$$
\sigma(t)=\varphi_{\sigma}(\log t) \leq \frac{1}{j} f(t)+\frac{D_{j}}{j}
$$

where $f(t):=h^{*}(\max \{0, \log (t)\})$. Hence, $\sigma(t)=o(f(t))$ as $t \rightarrow \infty$, and putting $f^{r}(t):=f\left(t^{r}\right)$ we have $\sigma^{r}(t)=o\left(f^{r}(t)\right)$.

Let us apply Lemma 4.4 to $\sigma^{r}, \omega^{r}$, and $f^{r}$ (instead of $\sigma, \omega$, and $f$ in the lemma). We obtain weights $\widetilde{\sigma}, \widetilde{\omega} \in \mathcal{W}$ satisfying $\gamma(\widetilde{\sigma}, \widetilde{\omega})>1$ and

$$
\omega^{r}(t)=o(\widetilde{\omega}(t)), \quad \sigma^{r}(t)=o(\widetilde{\sigma}(t)), \quad \tilde{\sigma}(t)=o\left(f^{r}(t)\right) \quad \text { as } t \rightarrow \infty .
$$

Hence, in view of (2), we have weights $\widetilde{\sigma}^{1 / r}, \widetilde{\omega}^{1 / r} \in \mathcal{W}$ such that

$$
\begin{gathered}
\gamma\left(\widetilde{\sigma}^{1 / r}, \widetilde{\omega}^{1 / r}\right)>r, \\
\omega(t)=o\left(\widetilde{\omega}^{1 / r}(t)\right), \quad \sigma(t)=o\left(\widetilde{\sigma}^{1 / r}(t)\right), \quad \widetilde{\sigma}^{1 / r}(t)=o(f(t)) \quad \text { as } t \rightarrow \infty .
\end{gathered}
$$

In particular, there is a constant $B>0$ such that $\tilde{\sigma}^{1 / r} \leq f+B$, whence for all $t \geq 0$

$$
\varphi_{\widetilde{\sigma}^{1 / r}}(t)=\widetilde{\sigma}^{1 / r}\left(e^{t}\right) \leq f\left(e^{t}\right)+B=h^{*}(t)+B
$$

and so (since $h$ is convex)

$$
g \leq h=h^{* *} \leq \varphi_{\widetilde{\sigma}^{1 / r}}^{*}+B .
$$

By the definition of $g$, we find $a \in \Lambda_{\left\{\widetilde{\sigma}^{1 / r}\right\}}=\Lambda_{\left\{\widetilde{\Sigma}^{1 / r}\right\}}$, where $\widetilde{\Sigma}^{1 / r}:=\mathcal{M}\left(\widetilde{\sigma}^{1 / r}\right)$, which is equivalent to

$$
\widehat{a} \in \Lambda_{\left\{\widehat{\left.\Sigma^{1 / r}\right\}}\right.} .
$$

By (13), we can apply Theorem 4.2 to $\widetilde{\omega}^{1 / r}$ and $\widetilde{\sigma}^{1 / r}$ (and $\gamma=r$ ) and conclude

$$
\widehat{a} \in \mathcal{B}\left(\mathcal{A}_{\left\{\widehat{\left.\Omega^{1 / r}\right\}}\right.}\left(S_{r}\right)\right),
$$

where $\widetilde{\Omega}^{1 / r}:=\mathcal{M}\left(\widetilde{\omega}^{1 / r}\right)$. By (14) and Lemma 4.5, $\mathcal{A}_{\left\{\widetilde{\left.\Omega^{1 / r}\right\}}\right.}\left(S_{r}\right) \subseteq \mathcal{A}_{(\widehat{\Omega})}\left(S_{r}\right)$ which gives the assertion because $\gamma<r$.

(ii) Fix $r>0$ such that $\gamma<r<\gamma(\sigma, \omega)$. Then $\gamma\left(\sigma^{r}, \omega^{r}\right)>1$ as above. The assumption $\sigma(t)=o(\tau(t))$ gives $\sigma^{r}(t)=o\left(\tau^{r}(t)\right)$ as $t \rightarrow \infty$. Applying Lemma 4.4 to $\sigma^{r}, \omega^{r}$, and $\tau^{r}$ (instead of $\sigma, \omega$, and $f$ in the lemma) and repeating the steps that led to (13) and (14), yields weight functions $\widetilde{\omega}^{1 / r}, \widetilde{\sigma}^{1 / r} \in \mathcal{W}$ satisfying (13) and

$$
\omega(t)=o\left(\widetilde{\omega}^{1 / r}(t)\right), \quad \sigma(t)=o\left(\widetilde{\sigma}^{1 / r}(t)\right), \quad \tilde{\sigma}^{1 / r}(t)=o(\tau(t)) \quad \text { as } t \rightarrow \infty .
$$

By Theorem 4.2 there exists $k_{0}>0$ such that for all $x>0$ and $h>0$ we have an extension operator

$$
\Lambda_{\left(\widetilde{\widetilde{S}^{1 / r}}\right)^{[x]}, h} \rightarrow \mathcal{A}_{\left(\widetilde{W^{1 / r}}\right)^{[8 x]}, k_{0} h}\left(S_{r}\right),
$$

where $\widetilde{\widetilde{\Sigma}^{1 / r}}=\left\{\left(\widehat{\widetilde{S}^{1 / r}}\right)^{[x]}: x>0\right\}$ and $\widetilde{\Omega^{1 / r}}=\left\{\left(\widehat{\widetilde{W}^{1 / r}}\right)^{[x]}: x>0\right\}$. By (15), Lemma 4.5, and Section 3.4, we have continuous inclusions

$$
\mathcal{A}_{\left(\widetilde{W^{1 / r}}\right)^{[8 x]}, k_{0} h}\left(S_{r}\right) \hookrightarrow \mathcal{A}_{\left\{\widetilde{\left.\Omega^{1 / r}\right\}}\right.}\left(S_{r}\right) \hookrightarrow \mathcal{A}_{(\widehat{\Omega})}\left(S_{r}\right)=\mathcal{A}_{\left(\tau_{2}\right)}\left(S_{r}\right) .
$$

Let $T:=\mathcal{M}(\tau)$. We have the continuous inclusions $\Lambda_{\{T\}}=\Lambda_{\{\tau\}} \subseteq \Lambda_{\left(\widetilde{\sigma}^{1 / r}\right)}=$

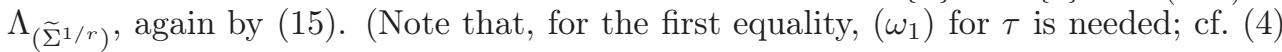
and Proposition 3.1). Furthermore, the linear mappings $\Lambda_{\{T\}} \rightarrow \Lambda_{\{\widehat{T}\}}$ and $\Lambda_{\left(\widetilde{\Sigma}^{1 / r}\right)} \rightarrow$ 
$\Lambda_{\left(\widetilde{\left.\Sigma^{1 / r}\right)}\right.}$ given by $a=\left(a_{p}\right) \mapsto \widehat{a}=\left(p ! a_{p}\right)$ are topological isomorphisms with inverse $\left(\widehat{a}_{p}\right) \mapsto\left(\widehat{a}_{p} / p !\right)$. Hence, the inclusion $\Lambda_{\left\{\tau_{3}\right\}}=\Lambda_{\{\widehat{T}\}} \subseteq \Lambda_{\left(\widetilde{\Sigma^{1 / r}}\right)}$ is continuous. Then the composite

$$
\Lambda_{\left\{\tau_{3}\right\}} \hookrightarrow \Lambda_{\left(\widetilde{\left.\widetilde{\Sigma}^{1 / r}\right)}\right.} \hookrightarrow \Lambda_{\left(\widetilde{\widetilde{S}^{1 / r}}\right)^{[x]}, h} \rightarrow \mathcal{A}_{\left(\widehat{W^{1 / r}}\right)^{[8 x]}, k_{0} h}\left(S_{r}\right) \hookrightarrow \mathcal{A}_{\left(\tau_{2}\right)}\left(S_{r}\right) \hookrightarrow \mathcal{A}_{\left(\tau_{2}\right)}\left(S_{\gamma}\right)
$$

is the required extension operator. The proof is complete.

4.6. Extensions controlled by the order of quasianalyticity. It is possible to have extensions on sectors of opening up to $\pi \mu(\omega)$, if one permits that $\sigma$ depends on the opening. We shall see that this is a consequence of Theorem 4.1. For a Roumieu version see [11, Theorem 6].

Theorem 4.6. Let $\omega \in \mathcal{W}$ Then:

(i) For any $0<r<\mu(\omega)$ there exists $\sigma \in \mathcal{W}$ such that for all $0<\gamma<r$ we have

$$
\mathcal{B}\left(\mathcal{A}_{\left(\tau_{2}\right)}\left(S_{\gamma}\right)\right) \supseteq \Lambda_{\left(\tau_{1}\right)},
$$

where $\tau_{1}:=\omega(\widehat{\mathcal{M}(\sigma)})$ and $\tau_{2}:=\omega(\widehat{\mathcal{M}(\omega)})$.

(ii) If $\tau \in \mathcal{W}$ satisfies $\sigma(t)=o(\tau(t))$ as $t \rightarrow \infty$, then there exists an extension operator

$$
\mathcal{E}^{\tau_{3}, \tau_{2}}: \Lambda_{\left\{\tau_{3}\right\}} \longrightarrow \mathcal{A}_{\left(\tau_{2}\right)}\left(S_{\gamma}\right)
$$

where $\tau_{3}:=\omega(\widehat{\mathcal{M}(\tau)})$.

(iii) The weight function $\sigma$ is minimal (up to equivalence) among all $\tau \in \mathcal{W}$ satisfying $\tau \underline{\Omega} \omega$ and $(\tau, \omega)_{\gamma_{r}}$.

Remark 4.7. Of course, minimality refers to the relation $@$ which induces a partial ordering on the set of equivalence classes of weight functions. The corresponding function (or sequence) space is then maximal; cf. Section 3.4 .

Proof of Theorem 4.6. Note that $\mu(\omega)>0$, by Section 2.4. For $0<r<\mu(\omega)$ we consider the weight function $\kappa_{\omega^{r}}^{1 / r}(t)=\kappa_{\omega^{r}}\left(t^{1 / r}\right)$, where

$$
\kappa_{\omega}(t):=\int_{1}^{\infty} \frac{\omega(t y)}{y^{2}} d y=t \int_{t}^{\infty} \frac{\omega(y)}{y^{2}} d y .
$$

Then $\left(\kappa_{\omega^{r}}^{1 / r}, \omega\right)_{\gamma_{r}}$ is valid by definition. The weight function $\kappa_{\omega^{r}}^{1 / r}$ has all properties defining $[\mathcal{W}$ except normalization which however can be achieved by switching to an equivalent weight, say $\sigma$, by redefining $\kappa_{\omega^{r}}^{1 / r}$ near 0; see [1, Remark 1.2 (b)] and [3, Remark 3.2(b)]. Then $\gamma(\sigma, \omega) \geq r>\gamma$ and so the statement follows from Theorem 4.1 The minimality of $\sigma$ is immediate from its definition and the relation $(\sigma, \omega)_{\gamma_{r}}$. Cf. [11, p.1650].

\section{Applications to the Weight sequence setting}

In this section we apply the extension results for weight functions to classes defined by weight sequences.

5.1. Mixed growth index $\gamma(M, N)$. Cf. [11, Section 3.1] and references therein. For a weight sequence $M$ and $r>0$ we consider the condition

$$
\sup _{p \in \mathbb{N}>0} \frac{\left(\mu_{p}\right)^{1 / r}}{p} \sum_{k \geq p}\left(\frac{1}{\mu_{k}}\right)^{1 / r}<\infty .
$$

It is immediate that $M$ satisfies $\left(\gamma_{r}\right)$ if and only if $M^{1 / r}$ satisfies $\left(\gamma_{1}\right)$. 
For weight sequences $M, N$ such that $\mu / \nu$ is bounded, consider the condition $(M, N)_{\gamma_{r}}$

$$
\sup _{p \in \mathbb{N}_{>0}} \frac{\left(\mu_{p}\right)^{1 / r}}{p} \sum_{k \geq p}\left(\frac{1}{\nu_{k}}\right)^{1 / r}<\infty .
$$

The mixed growth index is defined by

$$
\gamma(M, N):=\sup \left\{r>0:(M, N)_{\gamma_{r}} \text { is satisfied }\right\}
$$

and $\gamma(M, N):=0$ if $(M, N)_{\gamma_{r}}$ holds for no $r>0$. Note that $\gamma(M):=\gamma(M, M)$ is the growth index used in [30]; see also [6, 7].

Remark 5.1. Let $M \in \mathcal{L C}$ be given.

(i) $M$ satisfies $\beta_{3}$ if and only if $\gamma(M)>0$; this follows from [6, Theorem 3.11 (v) $\Leftrightarrow$ (vii)] applied to $\beta=0$.

(ii) We have $\gamma\left(\omega_{M}\right) \geq \gamma(M)$ and equality holds if $M$ has moderate growth; see [6. Corollary 4.6].

(iii) $\omega_{M}$ satisfies ( $\omega_{1}$ ) if and only if $\gamma\left(\omega_{M}\right)>0$; see [6, Corollary 2.14]. So, if $M \in \mathcal{L C}$ has moderate growth, then $\omega_{M}$ satisfies $\left(\omega_{1}\right)$ (i.e., $\omega_{M} \in \mathcal{W}$ ) if and only if $M$ satisfies $\beta_{3}$. In general, for a sequence $N \in \mathcal{L C}$ (not necessarily having moderate growth), $\omega_{N}$ has the property $\left(\omega_{1}\right)$ if and only if

$$
\exists L \in \mathbb{N}_{>0}: \liminf _{p \rightarrow \infty} \frac{\left(N_{L p}\right)^{1 /(L p)}}{\left(N_{p}\right)^{1 / p}}>1,
$$

as it is shown in [27, Theorem 3.1].

(iv) These statements are consistent with the implication [2, Lemma 12, (2) $\Rightarrow(4)]$.

5.2. Extensions controlled by the mixed growth index. The following theorem is a Beurling version of [11, Theorem 4].

Theorem 5.2. Let $M, N \in \mathbb{\mathcal { L C }}$ be such that $\mu / \nu$ is bounded, $M$ has moderate growth, and $\omega_{M}, \omega_{N} \in \mathcal{W}$, Then:

(i) $\gamma(M, N)=\gamma\left(\omega_{M}, \omega_{N}\right)>0$.

(ii) For any $0<\gamma<\gamma(M, N)$ we have the inclusion $\mathcal{B}\left(\mathcal{A}_{(\widehat{N})}\left(S_{\gamma}\right)\right) \supseteq \Lambda_{(\widehat{M})}$.

(iii) Let $L \in \mathcal{L C}$ satisfy $L \square M$ and assume that $\omega_{L} \in \mathcal{W}$. Then there exists an extension operator

$$
\mathcal{E}^{L, N}: \Lambda_{\{\widehat{L}\}} \longrightarrow \mathcal{A}_{(\widehat{N})}\left(S_{\gamma}\right) .
$$

Proof. (i) By [11, Lemma 4] we have $\gamma(M, N)=\gamma\left(\omega_{M}, \omega_{N}\right)$. Condition ( $\left.\omega_{1}\right)$ for $\omega_{N}$ yields $\gamma\left(\omega_{N}\right)>0$ (see Remark 5.1) and so $\gamma\left(\omega_{M}, \omega_{N}\right) \geq \gamma\left(\omega_{N}\right)>0$ (by Section 2.3).

(ii) Let $\Omega:=\mathcal{M}\left(\omega_{N}\right)$ and $\Sigma:=\mathcal{M}\left(\omega_{M}\right)$. Since $M$ has moderate growth, all sequences in $\Sigma=\left\{S^{[x]}: x>0\right\}$ are equivalent (see Lemmas 2.2 and 2.3), hence the same holds for $\widehat{\Sigma}$. The proof of [26, Theorem 6.4] yields $S^{[1]}=M$, hence $\widehat{S}^{[1]}=\widehat{M}$ and so $\Lambda_{(\widehat{M})}=\Lambda_{\left(\widehat{S}^{[1]}\right)}=\Lambda_{(\widehat{\Sigma})}=\Lambda_{\left(\tau_{1}\right)}$ for the weight function $\tau_{1}=\omega_{\widehat{M}} \in \mathcal{W}$. By Theorem 4.1 applied to to $\omega_{M}$ and $\omega_{N}$, we conclude, for $\tau_{2}=\omega(\widehat{\Omega}) \in \mathcal{W}$

$$
\Lambda_{(\widehat{M})}=\Lambda_{\left(\tau_{1}\right)} \subseteq \mathcal{B}\left(\mathcal{A}_{\left(\tau_{2}\right)}\left(S_{\gamma}\right)\right)=\mathcal{B}\left(\mathcal{A}_{(\widehat{\Omega})}\left(S_{\gamma}\right)\right) \subseteq \mathcal{B}\left(\mathcal{A}_{(\widehat{N})}\left(S_{\gamma}\right)\right) ;
$$

the last inclusion is clear by the definition of the classes and since $\widehat{N}=\widehat{W}^{[1]} \in \widehat{\Omega}$.

(iii) The relation $L \varangle M$ implies, by the definition of associated weight functions,

$$
\forall A \geq 1 \exists C \geq 1 \forall t \geq 0: \omega_{M}(A t) \leq \omega_{L}(t)+C .
$$

In combination with the fact that $\omega_{M}$ satisfies $\left(\sqrt{\omega_{6}}\right)$, since $M$ has moderate growth (see Lemma 2.2), we infer that $\omega_{M}(t)=o\left(\omega_{L}(t)\right)$ as $t \rightarrow \infty$. Now it suffices to apply Theorem 4.1(ii) to $\omega_{L}, \omega_{M}$, and $\omega_{N}$ (instead of $\tau, \sigma$, and $\omega$ ) and to note that $\mathcal{A}_{\left(\tau_{2}\right)}\left(S_{\gamma}\right) \hookrightarrow \mathcal{A}_{(\widehat{N})}\left(S_{\gamma}\right)$ and $\Lambda_{\{\widehat{L}\}} \hookrightarrow \Lambda_{\left\{\widehat{\mathcal{M}\left(\omega_{L}\right)}\right\}}$; see Section 3.4 
Example 5.3. Here is an explicit example of sequences which fulfill the assumptions of Theorem 5.2 and underline its value: Let $\gamma>\gamma^{\prime}>1$. By [11, Lemma 13, Theorem 7], there exist sequences $M, M^{\prime} \in \mathcal{L C}$ having moderate growth such that

- $p^{\gamma} \leq \mu_{p} \leq p^{\gamma(2 \gamma-1)}$ and $p^{\gamma^{\prime}} \leq \mu_{p}^{\prime} \leq p^{\gamma^{\prime}\left(2 \gamma^{\prime}-1\right)}$ for all $p \in \mathbb{N}$,

- $\gamma(M)=\gamma\left(M^{\prime}\right)=0$.

For $\varepsilon>0$ set $M_{\varepsilon}:=\left(p !^{\varepsilon} M_{p}\right)$ and $M_{\varepsilon}^{\prime}:=\left(p !^{\varepsilon} M_{p}^{\prime}\right)$. Then $\gamma\left(M_{\varepsilon}\right)=\gamma\left(M_{\varepsilon}^{\prime}\right)=\varepsilon>0$ (see [6. Theorem 3.11]) and thus $\omega_{M_{\varepsilon}}$ and $\omega_{M_{\varepsilon}^{\prime}}$ satisfy ( $\omega_{11}$ ) (see Remark 5.11). By construction, $M_{\varepsilon}$ and $M_{\varepsilon}^{\prime}$ have moderate growth. If we additionally assume that

$$
\gamma^{\prime}\left(2 \gamma^{\prime}-1\right) \leq \gamma,
$$

then $\mu_{\varepsilon}^{\prime} \leq \mu_{\varepsilon}$ and $\left(M_{\varepsilon}^{\prime}, M_{\varepsilon}\right)_{\gamma_{r}}$, for all $0<r<\gamma$, thus $\gamma\left(M_{\varepsilon}^{\prime}, M_{\varepsilon}\right) \geq \gamma$. So Theorem 5.2 can be applied to $M_{\varepsilon}^{\prime}$ and $M_{\varepsilon}$. Note that, by choosing $\gamma$ and $\varepsilon$ appropriately, one can make $\gamma\left(M_{\varepsilon}^{\prime}, M_{\varepsilon}\right)=\gamma\left(\omega_{M_{\varepsilon}^{\prime}}, \omega_{M_{\varepsilon}}\right)$ arbitrarily large and $\gamma\left(M_{\varepsilon}\right)=\gamma\left(M_{\varepsilon}^{\prime}\right)=\gamma\left(\omega_{M_{\varepsilon}}\right)=$ $\gamma\left(\omega_{M_{\varepsilon}^{\prime}}\right)>0$ arbitrarily small.

5.3. Order of quasianalyticity $\mu(N)$. In analogy to Section 4.6 we consider the order of quasianalyticity for a weight sequence $N \in \mathcal{L C C}$ (see [11, Section 3.2]):

$$
\mu(N):=\sup \left\{r>0: \sum_{k \geq 1}\left(\frac{1}{\nu_{k}}\right)^{1 / r}<\infty\right\}=\sup \left\{r>0: N \text { satisfies }\left[\overline{n \mathrm{q}_{r}}\right\}\right\}
$$

and $\mu(N):=0$ if $\left(\overline{\mathrm{nq}_{r}}\right)$ holds for no $r>0$. Note that $\mu(N)^{-1}$ coincides with the exponent of convergence of $N$; cf. [24, Prop. 2.13, Def. 3.3, Thm. 3.4] and [7, p.145]. If $M \in \mathbb{L C}$ is equivalent to $N$, then $\mu(M)=\mu(N)$.

5.4. Descendant construction. We recall a construction from [11, Remark 9], based on [20, Section 4.1]. Let $N \in[\mathcal{L C}$ be non-quasianalytic and $r>0$. The descendant of $N^{1 / r}$ is the sequence $S=S(N, r)$ defined by $S_{p}=\sigma_{0} \sigma_{1} \cdots \sigma_{p}$, where $\sigma_{0}:=1$ and

$$
\sigma_{p}:=\frac{\tau_{1} p}{\tau_{p}}, \quad \tau_{p}:=\frac{p}{\left(\nu_{p}\right)^{1 / r}}+\sum_{j \geq p}\left(\frac{1}{\nu_{j}}\right)^{1 / r}, \quad p \geq 1 .
$$

Notice that $S \in \overline{\mathcal{L C}}$ is strongly log-convex, see [20, Lemma 4.2]; for more of its properties we refer to [11, Remark 9]. For us the sequence $L=L(N, r) \in[\mathcal{L C}$ defined by

$$
L:=S^{r}
$$

is crucial. We have $L_{p}=\lambda_{0} \lambda_{1} \cdots \lambda_{p}$ with $\lambda:=\sigma^{r}$. It has the following properties (see [11, Remark 9, Lemma 6]):

(i) $(L, N)_{\gamma_{r}}$ and thus $\gamma(L, N) \geq r$.

(ii) $\lambda / \nu$ is bounded and so $(L, N)_{\gamma_{r^{\prime}}}$ for all $0<r^{\prime} \leq r$.

(iii) If $M \in \mathcal{L C C}$ satisfies $(M, N)_{\gamma_{r}}$ and $\mu / \nu$ is bounded, then also $\mu / \lambda$ is bounded. Consequently, $L$ is maximal (up to multiplication of $\lambda$ by a constant) among all sequences $M$ with $(M, N)_{\gamma_{r}}$ and $\mu / \nu$ bounded.

(iv) $L$ has moderate growth if and only if

$$
\exists C \geq 1 \forall k \in \mathbb{N}_{>0}: \frac{\left(\nu_{2 k}\right)^{1 / r}}{\left(\nu_{k}\right)^{1 / r}} \leq C+C \frac{\left(\nu_{2 k}\right)^{1 / r}}{2 k} \sum_{j \geq 2 k} \frac{1}{\left(\nu_{j}\right)^{1 / r}} .
$$

Moderate growth for $N^{1 / r}$ (equivalently for $N$ ), implies (17). In general, the converse implication is not true; see [11, Example 1]. 
5.5. Extensions controlled by the order of quasianalyticity. Now we are ready to prove a Beurling version of [11, Theorem 5].

Theorem 5.4. Let $N \in \mathcal{L C}$ satisfy $\sqrt{\left.\beta_{3}\right)}$. Then $\mu(N)>0$. Let $0<r<\mu(N)$ and suppose that (17) holds true for this value $r$. Then there exists $L \in \mathcal{L C}$ having moderate growth and with the following properties:

(i) $\mathcal{B}\left(\mathcal{A}_{(\widehat{N})}\left(S_{\gamma}\right)\right) \supseteq \Lambda_{(\widehat{L})}$ for each $0<\gamma<r$.

(ii) If $M \in \mathcal{L C}$ satisfies $M \square \mathbb{L}$ and $\omega_{M} \in \mathcal{W}$, then there is an extension operator

$$
\mathcal{E}^{M, N}: \Lambda_{\{\widehat{M}\}} \rightarrow \mathcal{A}_{(\widehat{N})}\left(S_{\gamma}\right) \text {. }
$$

(iii) $L$ is maximal among all $M \in \mathcal{L C}$ with $(M, N)_{\gamma_{r}}$ and $\mu / \nu$ bounded.

Proof. We have $\mu(N) \geq \gamma(M, N) \geq \gamma(N)$ for any sequence $M \in \mathcal{L C}$ with $\mu / \nu$ bounded, see [11, Lemmas 3, 5, Remark 8], and $\gamma(N)>0$, by Remark [5.1. Hence $\mu(N)>0$.

For $r$ as in the assumption, let $L=L(N, r)$ be the sequence defined in (16). Then $L$ has moderate growth, $\gamma(L, N) \geq r, \lambda / \nu$ is bounded, and $L$ satisfies (iii), by the properties listed in Section 5.4 Furthermore, $S=L^{1 / r}$ is strongly log-convex and hence satisfies $\left.\beta_{3}\right)$. Consequently, by Remark $5.1\left(\right.$ (iii), $\omega_{S}$ satisfies ( $\left.\omega_{1}\right)$. In view of (16) and (3), also $\omega_{L}$ has the property (|w1), and so $\omega_{L} \in \mathcal{W}$ Remark [5.1 also implies that $\omega_{N} \in \mathcal{W}$. Thus Theorem 5.2 shows that $L$ satisfies (i) and (ii).

\section{REFERENCES}

[1] J. Bonet, R. W. Braun, R. Meise, and B. A. Taylor, Whitney's extension theorem for nonquasianalytic classes of ultradifferentiable functions, Studia Math. 99 (1991), no. 2, 155-184.

[2] J. Bonet, R. Meise, and S. N. Melikhov, A comparison of two different ways to define classes of ultradifferentiable functions, Bull. Belg. Math. Soc. Simon Stevin 14 (2007), 424-444.

[3] J. Bonet, R. Meise, and B. A. Taylor, On the range of the Borel map for classes of nonquasianalytic functions, North-Holland Mathematics Studies - Progress in Functional Analysis 170 (1992), 97-111.

[4] R. W. Braun, R. Meise, and B. A. Taylor, Ultradifferentiable functions and Fourier analysis, Results Math. 17 (1990), no. 3-4, 206-237.

[5] J. Chaumat and A.-M. Chollet, Surjectivitè de l'application restriction à un compact dans les classes de fonctions ultradifférentiables, Math. Ann. 298 (1994), 7-40.

[6] J. Jiménez-Garrido, J. Sanz, and G. Schindl, Indices of O-regular variation for weight functions and weight sequences, Rev. R. Acad. Cienc. Exactas Fís. Nat. Ser. A. Mat. RACSAM 113 (2019), no. 4, 3659-3697.

[7] _ Injectivity and surjectivity of the asymptotic Borel map in Carleman ultraholomorphic classes, J. Math. Anal. Appl. 469 (2019), no. 1, 136-168.

[8] - Sectorial extensions, via Laplace transforms, in ultraholomorphic classes defined by weight functions, Results Math. 74 (2019), no. 27.

[9] Sectorial extensions for ultraholomorphic classes defined by weight functions, Math. Nachr. 293 (2020), no. 11, 2140-2174.

[10] _ The surjectivity of the Borel mapping in the mixed setting for ultradifferentiable ramification spaces, Monatsh. Math. 191 (2020), no. 3, 537-576.

[11] Ultraholomorphic extension theorems in the mixed setting, Banach J. Math. Anal. 14 (2020), no. 4, 1630-1669.

[12] H. Komatsu, Ultradistributions. I. Structure theorems and a characterization, J. Fac. Sci. Univ. Tokyo Sect. IA Math. 20 (1973), 25-105.

[13] M. Langenbruch, Extension of ultradifferentiable functions, Manuscripta Math. 83 (1994), no. 2, 123-143.

[14] A. Lastra, S. Malek, and J. Sanz, Continuous right inverses for the asymptotic Borel map in ultraholomorphic classes via a Laplace-type transform, J. Math. Anal. Appl. 396 (2012), 724-740.

[15] _ Summability in general Carleman ultraholomorphic classes, J. Math. Anal. Appl. (2015), no. 430, 1175-1206.

[16] S. Mandelbrojt, Séries adhérentes, régularisation des suites, applications, Gauthier-Villars, Paris, 1952. 
[17] H.-J. Petzsche, On E. Borel's theorem, Math. Ann. 282 (1988), no. 2, 299-313.

[18] A. Rainer, On the extension of Whitney ultrajets of Beurling type, Results Math. 76, 36 (2021), https://doi.org/10.1007/s00025-021-01347-z

[19] A. Rainer and G. Schindl, Composition in ultradifferentiable classes, Studia Math. 224 (2014), no. 2, 97-131.

[20] — Extension of Whitney jets of controlled growth, Math. Nachr. 290 (2017), no. 14-15, $2356-2374$.

[21] _ On the extension of Whitney ultrajets, Studia Math. 245 (2019), no. 3, $255-287$.

[22] _ On the extension of Whitney ultrajets, II, Studia Math. 250 (2020), no. 3, $283-295$.

[23] J.-P. Ramis, Devissage Gevrey, Journees singulieres de Dijon, 12-16 juin 1978. Conferences organisees par l'équipe de topologie du Department de Mathématiques de l'Universite de Dijon, 1978, pp. 173-204 (English).

[24] J. Sanz, Flat functions in Carleman ultraholomorphic classes via proximate orders, J. Math. Anal. Appl. (2014), no. 415, 623-643.

[25] G. Schindl, Exponential laws for classes of Denjoy-Carleman-differentiable mappings, 2014, PhD Thesis, Universität Wien, available online at http://othes.univie.ac.at/32755/1/2014-01-26_0304518.pdf

[26] Characterization of ultradifferentiable test functions defined by weight matrices in terms of their Fourier transform, Note di Matematica 36 (2016), no. 2, 1-35.

[27] _ On subadditivity-like conditions for associated weight functions, 2021, available online at https://arxiv.org/pdf/2101.11411.pdf

[28] J. Schmets and M. Valdivia, Extension maps in ultradifferentiable and ultraholomorphic function spaces, Studia Math. 143 (2000), no. 3, 221-250.

[29] - On certain extension theorems in the mixed Borel setting, J. Math. Anal. Appl. 297 (2003), 384-403.

[30] V. Thilliez, Division by flat ultradifferentiable functions and sectorial extensions, Results Math. 44 (2003), 169-188.

Fakultät für Mathematik, Universität Wien, Oskar-Morgenstern-Platz 1, A-1090 Wien, Austria.

Email address: david.nicolas.nenning@univie.ac.at

Email address: armin.rainer@univie.ac.at

Email address: gerhard.schindl@univie.ac.at 